INPLASY

PROTOCOL

To cite: Chen et al. Effects of proton pump inhibitors in cancer patients treated with immune checkpoint inhibitors: a systematic review and metaanalysis. Inplasy protocol 202010088. doi:

10.37766/inplasy2020.10.0088

Received: 23 October 2020

Published: 23 October 2020

Corresponding author:

Baoqing Chen

chenbq@sysucc.org.cn

Author Affiliation:

Sun Yat-sen University Cancer Center

Support: NSFC(No. 81902462).

Review Stage at time of this submission: Preliminary searches.

Conflicts of interest: None.

\section{Effects of proton pump inhibitors in cancer patients treated with immune checkpoint inhibitors: a systematic review and meta-analysis}

\author{
Chen, BQ1'; Yang, C2; Li, QQ3; Dragomir, M4; Calin, GA5.
}

Review question / Objective: Whether the use of proton pump inhibitors (PPIs) in cancer patients who are treated with immune checkpoint inhibitors (ICIs), before and/or after ICls treatment initiation, affects the response rate, overall survival, and progression-free survival of these patients?

Condition being studied: Currently, immune checkpoint inhibitor (ICl) has achieved remarkable efficiency in disease control in certain types of cancers, especially melanoma, lung cancer, and microsatellite stable colon cancer. Although, ICls seem to be very effective they are limited by a low response rate.There is growing number of studies showing that altered gut microbiota can negatively impact homeostasis, systemic immune response, and ICls efficacy. Recent evidence indicates that PPIs can cause significant gut microbiota dysregulations. Furthermore, previous related studies showed contradictory results between detrimental and no effects on the survival of cancer patients treated with $\mathrm{ICl}$ and PPIs concomitantly. Therefore, to better define the effect of PPIs on cancer patients treated with ICls, we are performing this systematic review and meta-analysis.

INPLASY registration number: This protocol was registered with the International Platform of Registered Systematic Review and Meta-Analysis Protocols (INPLASY) on 23 October 2020 and was last updated on 23 October 2020 (registration number INPLASY2020100088).

\section{INTRODUCTION}

Review question / Objective: Whether the use of proton pump inhibitors (PPIs) in cancer patients who are treated with immune checkpoint inhibitors (ICIs), before and/or after ICls treatment initiation, affects the response rate, overall survival, and progression-free survival of these patients?

Condition being studied: Currently, immune checkpoint inhibitor (ICI) has achieved remarkable efficiency in disease control in 
certain types of cancers, especially melanoma, lung cancer, and microsatellite stable colon cancer. Although, ICls seem to be very effective they are limited by a low response rate.There is growing number of studies showing that altered gut microbiota can negatively impact homeostasis, systemic immune response, and ICls efficacy. Recent evidence indicates that PPIs can cause significant gut microbiota dysregulations. Furthermore, previous related studies showed contradictory results between detrimental and no effects on the survival of cancer patients treated with ICl and PPIs concomitantly. Therefore, to better define the effect of PPIs on cancer patients treated with ICls, we are performing this systematic review and meta-analysis.

\section{METHODS}

Search strategy: The search process will follow the Preferred Reporting Items for Systematic Reviews and Meta-analyses (PRISMA) guidelines. We will mainly search the EMBASE, MEDLINE (via PubMed), Cochrane Library databases, and major oncology conferences' (ASCO, ESMO, AACR, SITC) proceedings. The following keywords will be used as search terms: ('immune checkpoint inhibitors' or ICls or PD-1 or PD-L 1 or CTLA-4 or pembrolizumab or nivolumab or atezolizumab or avelumab or durvalumab or tremelimumab or ipilimumab or toripalimab or sintilimab or camrelizumab) and (proton pump inhibitors or PPIs or omeprazole or lansoprazole or dexlansoprazole or esomeprazole or pantoprazole or rabeprazole).

Participant or population: Cancer patients treated with immune checkpoint inhibitors (ICls) and receiving concomitantly systemic proton pump inhibitors (PPIs).

Intervention: 1. The included immune checkpoint inhibitors will be pembrolizumab, nivolumab, atezolizumab, avelumab, durvalumab, tremelimumab, ipilimumab, toripalimab, sintilimab, camrelizumab and combination of these. 2. Patients receiving ICls in combination with chemotherapy or targeted therapy will also be included in the study. 3. PPI use is defined as any PPI administration between $\mathbf{3 0}$ days prior and $\mathbf{3 0}$ days after treatment initiation.

Comparator: Cancer patients treated with ICls and not receiving concomitantly any PPI.

Study designs to be included: Prospective or retrospective studies.

Eligibility criteria: Prospective or retrospective studies (publications, abstracts, posters and trials), comparing overall survival (OS) and progression-free survival (PFS) between ICls plus PPIs use (intervention group) and ICls use alone (comparator group) in cancer patients will be selected. Exclusion criteria will be as follows: (1) reviews, editorials, comments, letters, and case reports; (2) studies with lack of information regarding a comparator group; (3) studies not reporting the survival outcome of patients treated with PPI; (4) insufficient data to extract hazard ratios (HRs) and 95\% confidence intervals (Cls).

Information sources: Publications that compared PPIs with non-PPIs use in cancer patients treated with ICIs will be identified by searching the EMBASE, MEDLINE (via PubMed), Cochrane Library databases, and major oncology conferences' (ASCO, ESMO, AACR, SITC) proceedings. We will manage to cover all available relevant studies (publications, abstracts, posters, and trials), and will not apply any filter on the language (although eventually be presented in English) or year of publication. At last, we will contact corresponding authors to find any missing data.

Main outcome(s): The main outcomes include the objective response rate(ORR) to ICIs, progression-free survival (PFS) and overall survival (OS). The measure of effect is hazard ratios(HR).

Data management: The data will be extracted independently by two authors. When discrepancies will occur, a third 
reviewer will analyze the study and decide on its inclusion in the systematic review and meta-analysis. One investigator will independently extract and summarize the data from all included studies using a standardized data extraction spreadsheet and the data will be reviewed by two other investigators. The following items will be extracted: -Source: country/region of origin, first author, year of publication, and type of study. -Patients and cancer characteristics: number of patients, types of cancer, cancer stage, comorbidities. -Treatment scheme/Types of Immune checkpoint inhibitors (ICls) -PPI Treatment: Types of PPIs, the reason for PPI intake, time and duration of using PPI, other side effects induced by PPI. -Results: median progression-free survival (PFS), median overall survival (OS), objective response rate(ORR), hazard ratio(HR) and $95 \%$ confidence interval $(95 \% \mathrm{Cl})$ or p-value for OS and PFS. (The HRs and $95 \%$ Cls from each study will be either extracted directly from original papers or will be calculated using Kaplan-Meier curves based on the method of Tierney et al.).

Quality assessment / Risk of bias analysis: Quality of paper will be evaluated using the Newcastle-Ottawa Scale (NOS) for observational studies and the Cochrane risk of bias assessment tool for randomized control trials. Whether a study is included will be determined based on the results of the rating. Two authors will independently evaluate the risk of bias. When a disagreement arises in the study rating, it will be resolved through discussion and consensus between all authors. Funnel plots with Egger's regression tests will be used to examine publication bias if there are more than 10 studies included in the meta-analysis.

Strategy of data synthesis: Data synthesis will be performed on Hazard Ratios (HR) for OS and PFS. Cochrane $Q$ tests and the $I^{2}$ index will be used to evaluate heterogeneity. If $\left.\right|^{2} \leq 50 \%$, we will apply a fixed-effect model to pool the data and calculate $95 \%$ confidence intervals. If heterogeneity is significant $\left(I^{2}>50 \%\right)$, a random-effect model will be used. Review
Manager (ver. 5.4) will be used for all pooled analyses. Stata (ver. 16.0) will be used for Egger's regression tests and sensibility analysis.

Subgroup analysis: The types of cancer and, time and duration of PPI treatment of participants are planned for subgroup analysis.

Sensibility analysis: Sensitivity analysis will be carried out for the results of metaanalysis if with obvious heterogeneity. We will add a scatter plot for sampling-based sensitivity analysis. Stata (ver.16.0) will be used for sensibility analysis.

Language: English.

Country(ies) involved: China, Germany and United States of America.

Keywords: proton pump inhibitors; cancer; immune checkpoint inhibitors; immunotherapy.

Contributions of each author:

Author 1 - Baoqing Chen.

Author 2 - Chen Yang.

Author 3 - Qiaoqiao Li.

Author 4 - Mihnea Dragomir.

Author 5 - George A Calin. 